\title{
Résumé d'un rapport destiné aux professionnels de la santé buccodentaire au Canada pour un retour sécuritaire à la pratique clinique pendant la pandémie de COVID-19
}

\author{
Bureau du dentiste en chef du Canada ${ }^{1}$
}

\section{Résumé}

Au début de la pandémie de la maladie à coronavirus 2019 (COVID-19), en mars 2020, les organismes de réglementation ont conseillé aux professionnels de la santé canadiens d'arrêter les soins de routine ou facultatifs et de ne fournir que des soins d'urgence. Deux mois plus tard, les gouvernements provinciaux et territoriaux ont mis en place des plans de "réouverture » de leurs régions. Les professionnels en santé buccodentaire fourniront toutefois des soins dans un environnement très différent que celui dans lequel ils travaillaient avant le début de la pandémie, et ce, principalement dans le domaine de la prévention des infections. Durant la pandémie de COVID-19, les décideurs dans le domaine de la santé buccodentaire de tous les niveaux ont pris des décisions et fourni des conseils et des directives, reposant souvent sur des renseignements imparfaits ou incomplets, dans un environnement très complexe et en évolution constante. Le Bureau du dentiste en chef du Canada a commandé l'élaboration d'un document d'évaluation national de haut niveau et multidisciplinaire, dans le domaine public, pour recueillir, résumer et présenter ces changements au niveau des environnements et protocoles de travail en santé buccodentaire. Ce document est accessible pour les organismes de réglementation, les formateurs, les responsables de programme et les décideurs politiques canadiens en santé buccodentaire.
Cette oeuvre est mise à la disposition selon les termes de la licence internationale Creative Commons Attribution 4.0

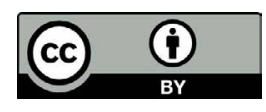

Affiliation

${ }^{1}$ Bureau du dentiste en chef du Canada, Agence de la santé publique du Canada, Ottawa, ON

\section{Correspondance :}

claudia.gorenko@canada.ca
Citation proposée : Bureau du dentiste en chef du Canada. Résumé d'un rapport destiné aux professionnels de la santé buccodentaire au Canada pour un retour sécuritaire à la pratique clinique pendant la pandémie de COVID-19. Relevé des maladies transmissibles au Canada 2020;46(11/12):421-2.

https://doi.org/10.14745/ccdr.v46i1112a01f

Mots-clés : santé buccodentaire, pratique clinique, post-pandémie, Canada, COVID-19

\section{Introduction}

Les divers leaders en santé buccodentaire (professionnels, organisationnels, institutionnels et cliniques) ainsi que les professionnels de la santé buccodentaire de première ligne, prennent des décisions quotidiennement pour être en mesure de mieux gérer les patients et de fournir une orientation, dans le cadre du retour à la pratique clinique durant la pandémie de maladie à coronavirus 2019 (COVID-19). Ces personnes et ces organisations prennent des décisions dans le cadre d'une crise très dynamique et dans un environnement changeant. Ces décisions reposent sur de nombreuses sources d'informations en évolution et ont une incidence dans toutes les régions du pays. Ces décisions sont prises à partir de directives fournies par des gouvernements ou d'autres personnes morales (comme les organismes de réglementation), reposant sur des données et des preuves scientifiques, sur l'opinion d'experts et sur des besoins priorisés. Ces décisions comportent des perspectives médicales, économiques, éthiques et autres, et les décideurs reconnaissent que les renseignements et les conseils sur lesquels reposent les décisions sont souvent imparfaits, incomplets ou limités. En résumé, les décideurs dans le domaine de la santé buccodentaire prennent des décisions et fournissent des orientations dans un environnement très complexe et en évolution rapide, souvent à partir de renseignements imparfaits ou incomplets.

L'on observe également que les professionnels de la santé buccale (dentistes, hygiénistes dentaires, aides-dentistes, denturologistes, techniciens dentaires et thérapeutes dentaires) dans l'ensemble du pays, dont la vaste majorité travaille dans des cabinets privés et non dans des installations publiques, ont été mandatés par leurs organismes de réglementation de cesser de fournir des soins de routine ou des soins facultatifs et de ne fournir que des soins d'urgence, au mois de mars 2020. En 
date du 4 mai 2020, le premier gouvernement provincial a mis en place un plan pour "rouvrir " sa région, y compris la santé buccodentaire. Les autres provinces et territoires ont rapidement fait de même. Les professionnels en santé buccodentaire retourneront toutefois prodiguer des soins dans un environnement très différent de celui dans lequel ils travaillaient avant le début de la pandémie, et ce, principalement dans le domaine de la prévention des infections. Le Bureau du dentiste en chef du Canada a demandé à I'Université McGill d'élaborer un document complet. À cet égard, le Bureau du dentiste en chef du Canada a convoqué un groupe multidisciplinaire fondé sur les connaissances, comprenant des représentants du domaine de la santé buccale à l'échelle nationale et des représentants du gouvernement fédéral. Un document unique de haut niveau à l'échelle nationale reposant sur les preuves actuelles a maintenant été généré et il appartient au domaine public (1). Les organismes de réglementation en santé buccale canadiens pourront ensuite choisir de consulter ce document lorsque vient le temps d'élaborer une orientation cohérente pour leurs titulaires au niveau provincial ou territorial. Les formateurs, les responsables de programme et les décideurs politiques peuvent également décider de consulter ce document, dans le cadre de leurs tâches respectives. Le dentiste en chef du Canada a transmis les lacunes en matière de donnée mises en lumière dans le cadre de ce processus aux Instituts de recherche en santé du Canada. II recommande de financer prioritairement ces domaines.

\section{Référence}

1. Bureau du dentiste en chef du Canada. Données probantes à l'appui d'un retour sécuritaire à la pratique clinique des professionnels en santé buccodentaire au Canada pendant la pandémie de COVID-19 : Rapport préparé pour le Bureau du dentiste en chef du Canada. www.canada.ca/fr/sante-publique/services/ma ladies/2019-nouveau-coronavirus/professionnels-sante/ donnees-probantes-retour-securitaire-pratique-clini que-sante-buccodentaire.html?utm_source=CCDR\&utm_ medium $=$ CCDR\&utm_campaign=McGill_report_covid_FRE 